\title{
Optical coherence tomography: the past, present and future
}

\author{
Raj K. Goel • Jihad H. Kaouk
}

Received: 29 August 2007 / Accepted: 12 September 2007 / Published online: 19 October 2007

(C) Springer London 2007

\begin{abstract}
Optical coherence tomography is a new technology that provides an atraumatic "optical biopsy" providing both anatomical and possible histopathologic results. It has only recently been incorporated into the urological field. This paper highlights previous and prospective advances of optical coherence tomography in urology and its potential application in robotic urologic surgery.
\end{abstract}

Keywords Optical coherence tomography - Laparoscopic . Prostate cancer $\cdot$ Prostatectomy $\cdot$ Robotic

\section{Introduction}

The search for atraumatic evaluation of tissue during surgery has led to the development of optical coherence tomography (OCT). An "optical biopsy" is a non-invasive method of providing anatomical and potentially histological evaluation through imaging.

Optical coherence tomography is an FDA-approved portable imaging system (Figs. 1 and 2) that provides real time histologic imaging. The system is analogous to ultrasound, however uses infrared light rather than acoustical waves and, unlike ultrasound, does not require direct contact with tissue or a transducing medium. The infra-red light is backscattered from the patient's tissue, collected by the probe's fiber, and, combined with a reference signal, produces a high spatial resolution image of the tissue microstructure. It acquires real-time images with $200 \times 200$ pixels, $15-\mu \mathrm{m}$ depth resolution in free space $(11 \mu \mathrm{m}$ in tissue), and $25 \mu \mathrm{m}$

R. K. Goel · J. H. Kaouk ( $\square)$

Cleveland Clinic, 9500 Euclid Avenue/A100,

Cleveland, OH 44195, USA

e-mail: kaoukj@ccf.org lateral resolution. Given that OCT is fiber-optic based, it allows for easy integration with endoscopes and catheters.

Optical coherence tomography was first applied in ophthalmology where it was used initially for transparent tissues of the eye. Since then, OCT has expanded its role to include macular disease, retinal pathology and corneal abnormalities $[1,2]$. Once OCT established itself in imaging delicate tissue, further expansion of its armamentarium to include solid organs was justified. Through technological modifications, "optical biopsies" of organs including gastrointestinal, cardiovascular, respiratory, skin, nervous system, and male and female reproductive organs have been performed [3-7].

The first application of optical coherence tomography in urology occurred in 1997 when in-vitro imaging of bladder, prostate, ureteral biopsies and neurovascular bundles were compared to $\mathrm{H}$ and $\mathrm{E}$ staining. As the first comparative study, high-resolution cross-sectional imaging obtained from OCT correlated well with the histological architecture of all the tissues sampled [8].

In this review, we highlight current and future applications of optical coherence tomography in the field of urology.

\section{Methods}

Through a comprehensive computer-based Medline search, MESH terms including optical coherence tomography and/ or laparoscopy, robotic, surgery, urology, bladder, prostate, cancer, and endoscopy were applied. All papers relevant to the use of optical coherence tomography in urological practice and published in English were included, such as case reports, and prospective, retrospective, and randomized studies. Basic science and animal research were also incorporated with, again, strict relevance to urological use. 
Fig. 1 Niris computer-based system with a laparoscopic probe

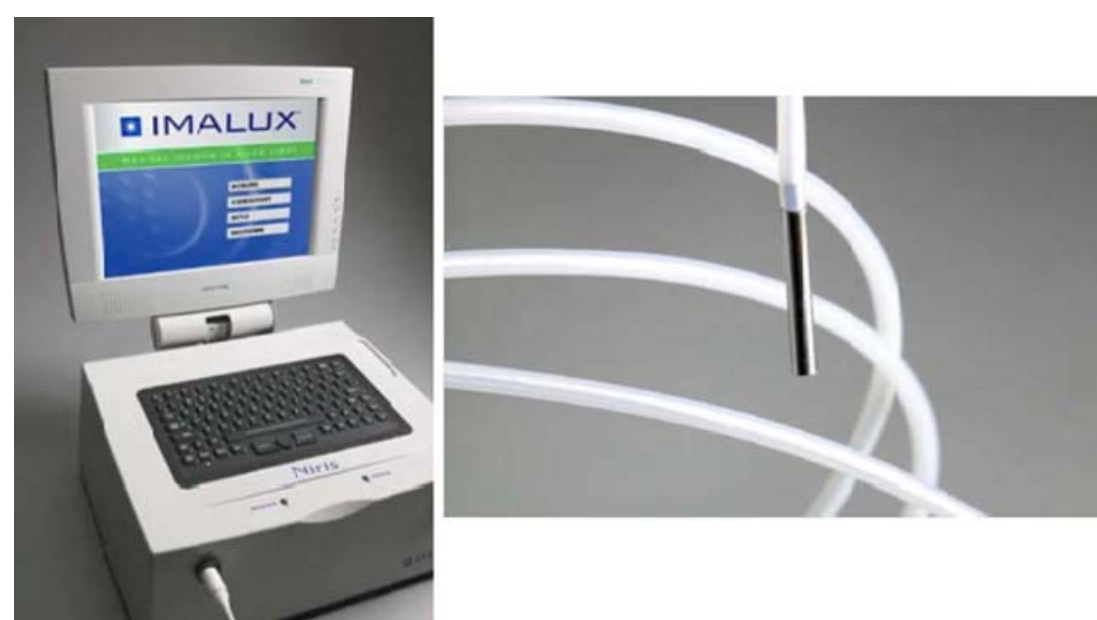

\section{Results}

A total of 18 journal articles evaluating optical coherence tomography and urology were evaluated. Nine papers were in relation to optical coherence tomography and urothelial abnormalities. The remaining journals focus primarily in OCT's role in the imaging characteristics of the prostate or prostate cancer. There is currently a solitary paper evaluating the use of optical coherence tomography in robotic urological practice.

\section{Bladder}

Urothelial malignancies are detected more commonly at a superficial stage and, although not aggressive, pose a significant challenge in monitoring due to high recurrence rates. Early detection and treatment of non-muscle invasive bladder cancer is paramount for bladder preservation and

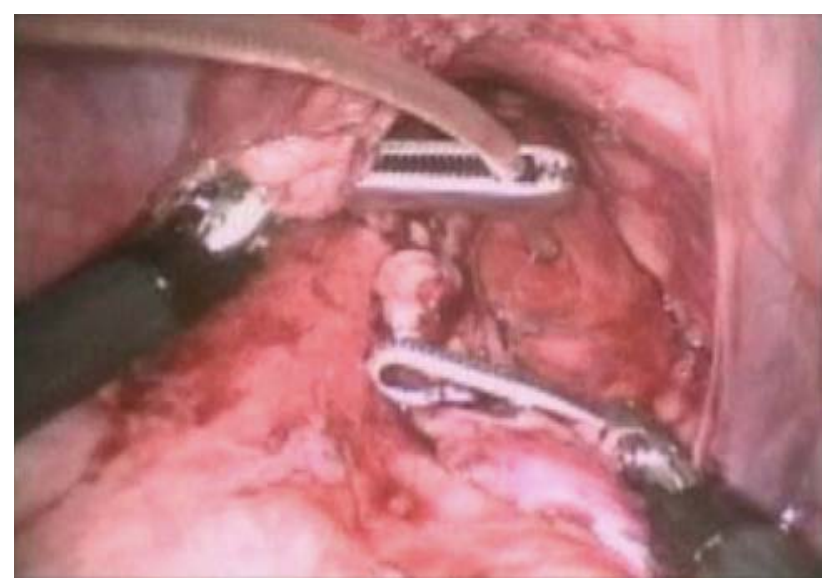

Fig. 2 Niris probe in vivo during animal robotic prostate surgery. Note the flexible OCT probe placed on the endopelvic fascia using the robotic articulating graspers longevity. Cystoscopy is considered the gold standard in the diagnosis and surveillance of urothelial carcinoma. However, diagnostic cystoscopy may overlook unapparent tumors, or falsely interpret malignant lesions as benign. Secondly, cystoscopy does not provide information on submucosal architecture to determine depth of tumor penetration once a lesion is identified.

An imaging modality that can provide near histopathological information can be an important diagnostic and prognostic tool for treating urothelial carcinoma. Jessner et al. [9] compared in-vitro OCT images to $\mathrm{H}$ and $\mathrm{E}$ analysis of human bladder tumor resection fragments. The tomography of muscle invasive urothelial carcinoma demonstrated architectural re-arrangement of the mucosal, submucosal, and muscularis boundaries in addition to individual muscle bundles. Manyak et al. [10] was able to address the in-vitro limitation of this study by imaging the urothelium in vivo. Twenty-four patients that were visually suspected of having urothelial carcinoma were scanned using OCT. Tomography was able to detect 29 of the 36 suspect areas in addition to discriminating between lamina propria and muscularis propria invasion. Overall, OCT proved to be $100 \%$ sensitive and $89 \%$ specific with a negative predictive value of $100 \%$. Although preliminary, OCT provides additional pathological information during diagnostic cystoscopy for the detection and staging of urothelial carcinoma.

Apart from malignancy, there are occasions where benign processes of the bladder are mistaken for malignancy. Unfortunately, these cases are diagnosed following a series of invasive bladder biopsies. Optical coherence tomography has demonstrated its ability to not only discern normal from malignant change, but also differentiate between proliferative and non-proliferative lesions of the bladder [11]. A few examples of optical coherence tomography of the bladder demonstrating carcinoma in situ are seen in Figs. 3a, b. 

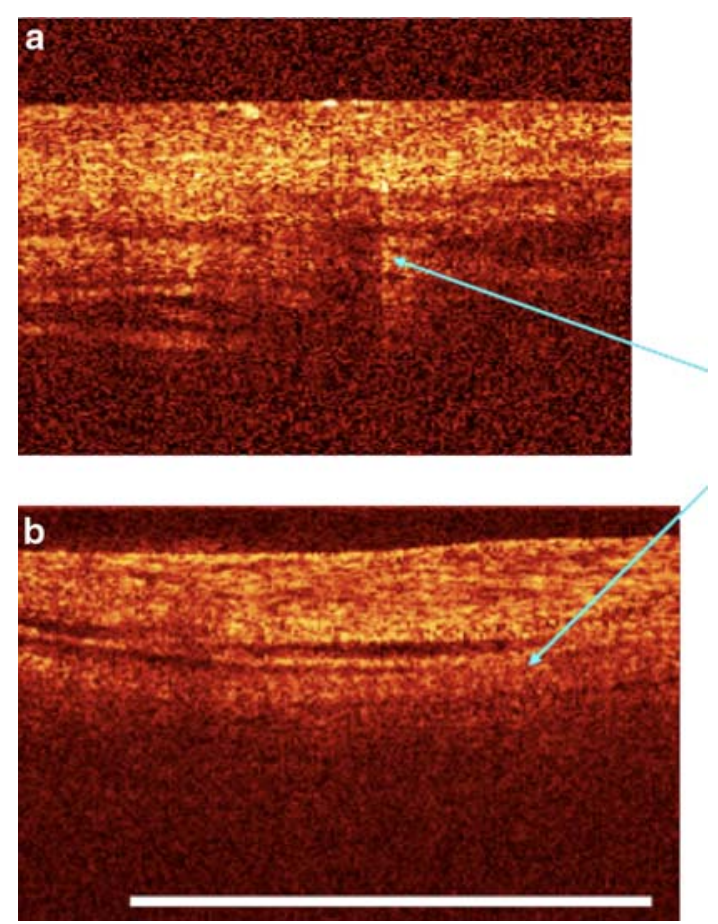

Fig. 3 a Optical coherence tomography of the bladder urothelium demonstrating carcinoma in situ. No clear urothelial layer is identified and the combined urothelium and lamina propria are bright. b Optical coherence tomography of the carcinoma in situ. Preserved horizontal layering indicates no invasion into the muscularis propria

\section{Prostate}

Prostate cancer has become the leading cancer diagnosed in men today. Although there are various modes of treatment of prostate cancer, radical prostatectomy has been shown to improve survival beyond 15 years [12]. Despite its overall survival advantage, radical prostatectomy carries inherent risks of incontinence and erectile dysfunction. Although anatomical nerve-sparing radical prostatectomy [13] has had a significant impact on postoperative potency, the overall outcomes do not meet both patient and surgeon expectations. During nerve-sparing radical prostatectomy, anatomical location of the neurovascular bundles is inferred on the basis of the vascular supply of the prostate. There is currently no established visual modality available to conclusively identify the neurovascular bundles during radical prostatectomy. However, Boppart et al. [14] has shown that optical coherence tomography can provide real-time invitro imaging during prostate surgery. Figures $4 \mathrm{a}$ and $4 \mathrm{~b}$ depict tomographical images of the prostate.

There are currently four methods of performing radical prostatectomy - the perineal, retropubic, laparoscopic, and robot-assisted techniques. With the expanded role of OCT, the potential use of in-vivo imaging of the neurovascular
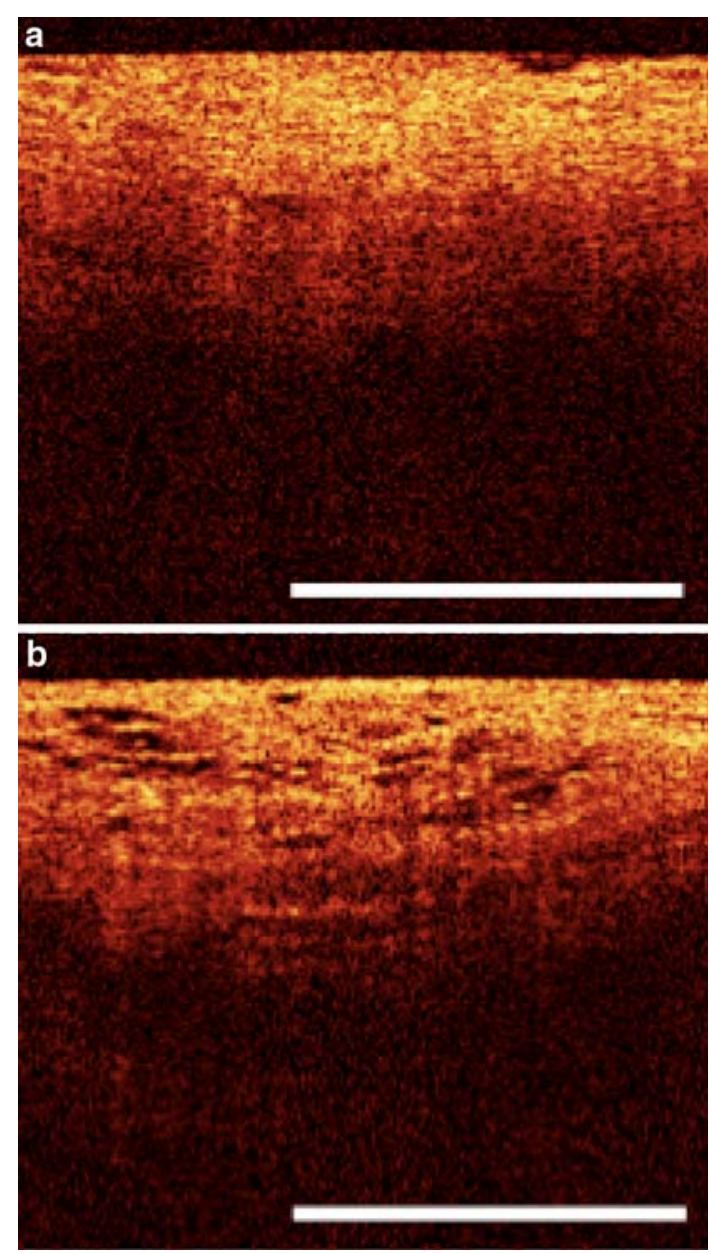

Fig. 4 a Surface tomography of the prostate demonstrating a dense fibromuscular stroma. b Surface tomography of the prostate showing a complex subcapsular venous network

bundles during radical prostatectomy is now real. At our institution, the first FDA-approved OCT imaging system for non-ophthalmologic use, Niris (Imalux, Cleveland, OH, USA) was used in vivo clinically [15]. The Niris system was applied to 24 patients that underwent either laparoscopic or robot-assisted radical prostatectomy. The eight French Niris probes can be inserted into the laparoscopic ports during surgery. Optical coherence tomography application during the procedure did not add to the operative time or morbidity to the patient. In an attempt to evaluate the feasibility of nerve-sparing prostatectomy, specimens were examined both in vivo and in vitro for the presence or absence of the neurovascular bundles. Optical coherence tomography was performed during the radical prostatectomy in an attempt to preserve the nerves using real-time data acquisition. Over 300 images using OCT were accumulated including those of the endopelvic fascia, fat, lymphatics, prostatic capsule, and neurovascular bundles. In four patients who underwent a wide excision of the 
neurovascular bundle, bench examination of the specimen proved a $100 \%$ correlation between the OCT image and the histological examination of the prostate. Figures $5 \mathrm{a}$ and $5 \mathrm{~b}$ demonstrate the characteristic images of the neurovascular bundles along the prostate.

More recently, Fried et al. [16] has used OCT's properties to identify the cavernousal nerves via an open rodent model. Six male Sprague-Dawley rats were anaesthetized and underwent open surgical exploration of the cavernous and ipsilateral major pelvic ganglion. Following identification of the neurovascular structures, simultaneous cavernousal nerve
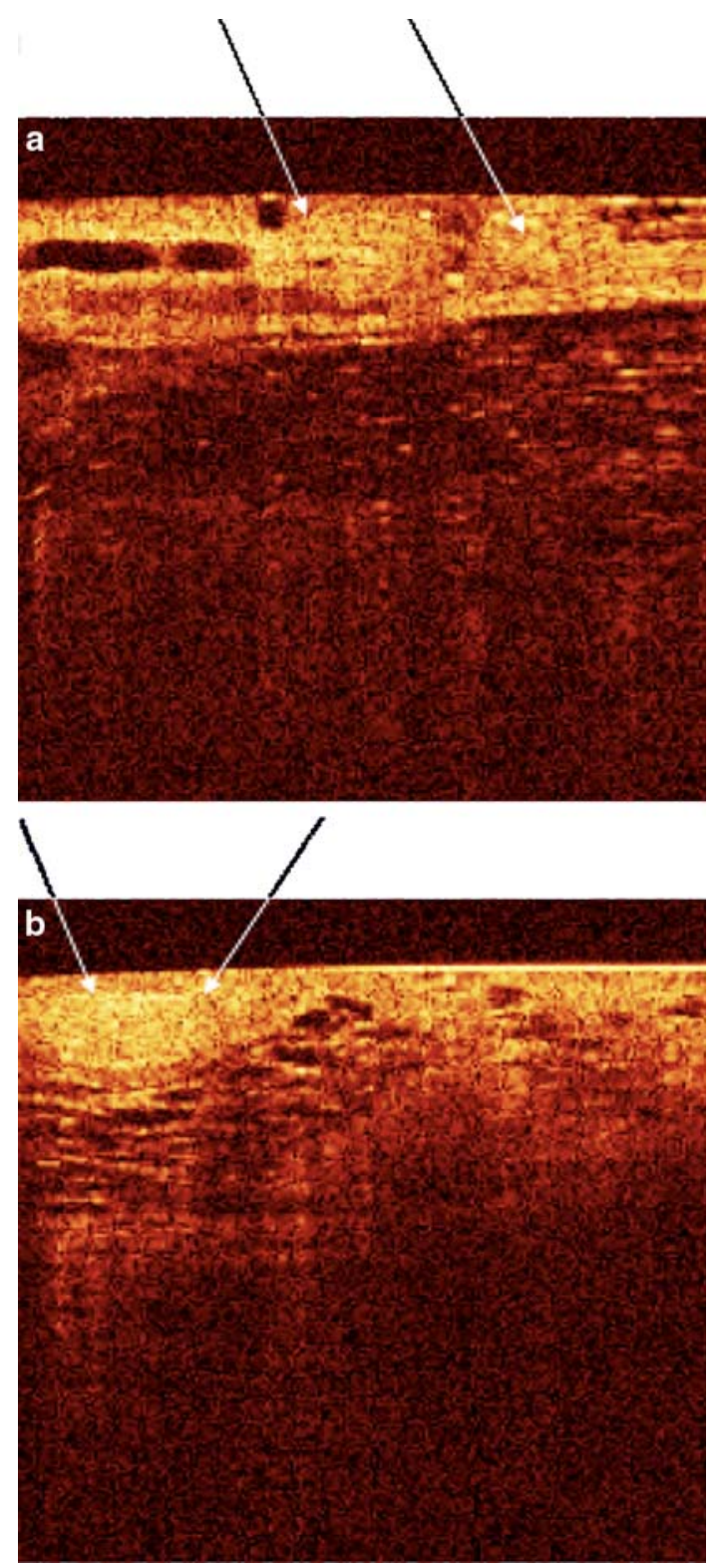

Fig. 5 a Optical coherence tomography of the neurovascular bundle along the prostate. $\mathbf{b}$ Cross-sectional tomography of a neurovascular bundle along the prostatic capsule stimulation and intracavernousal pressure monitoring was performed. This approach provided confirmation of the neurovascular bundle detected by OCT. A typical response achieved during $30 \mathrm{~s}$ of nerve stimulation resulted in a pressure increase from 10 to $80 \mathrm{mmHg}$. Following stimulation, these animals were euthanized to evaluate the OCT image in comparison to the histological appearance of the neurovascular bundle.

Examining the real-time optical coherence tomography during nerve-sparing radical prostatectomy will be the next logical step. By identifying the nerves prospectively with OCT, perhaps an improved functional outcome can result.

\section{Kidney}

It has been well established that partial nephrectomy has equivalent oncological results to radical nephrectomy in select patients [17]. The technique of performing partial nephrectomy can include laparoscopic or open approaches. The minimally invasive approach is technically demanding due to surgical resection and reconstruction of the kidney, usually within a limited time of warm ischemia. Optical coherence tomography and its use in laparoscopic partial nephrectomy have been explored at our institution. Chung et al. [18] incorporated the Niris system in eleven laparoscopic partial nephrectomies. Over 500 images were obtained during the surgical extirpation of the renal mass. Images of the parenchyma and the capsule both on and adjacent to the renal mass were obtained. Optical coherence tomography was able to detect structural abnormalities adjacent to and on the capsule of nine histologically confirmed renal cell carcinomas (Figs. 6a, b).

\section{Limitations and challenges}

A limitation of OCT is its shallow depth of penetration and image acquisition. Limited by its probe size and diameter, multiple data sites and angles are required to obtain an impression of the anatomical structure being observed. Further experience with OCT in conjunction with histological analysis will also aid the operator in discerning from various structures which demonstrate overlapping imaging characteristics. For example, lymphatic tissue looks very similar tomographically to neural tissue (Fig. 7).

\section{Conclusion}

The prospective benefit of optical coherence tomography is promising for operative urology. Although relatively 

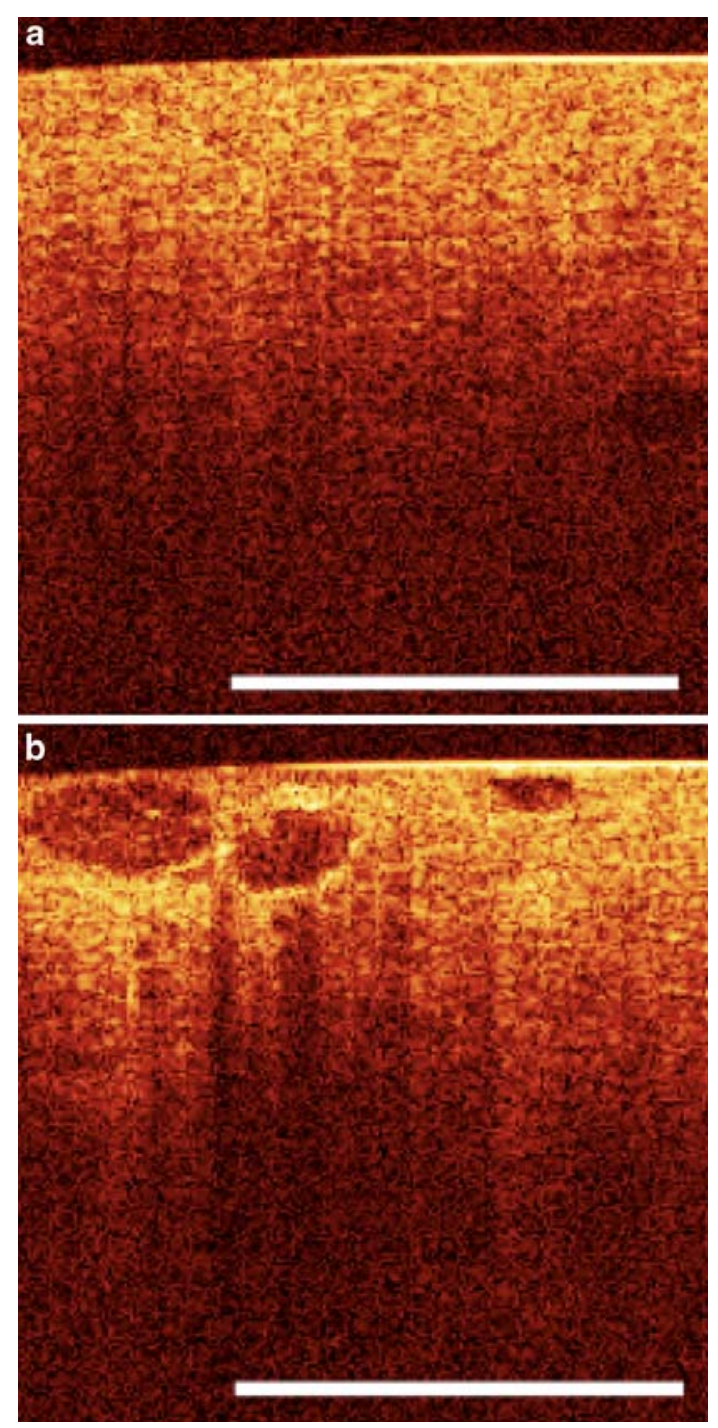

Fig. 6 a Optical coherence tomography of normal renal parenchyma. b Optical coherence tomography of renal parenchyma with a histological proven renal cell carcinoma

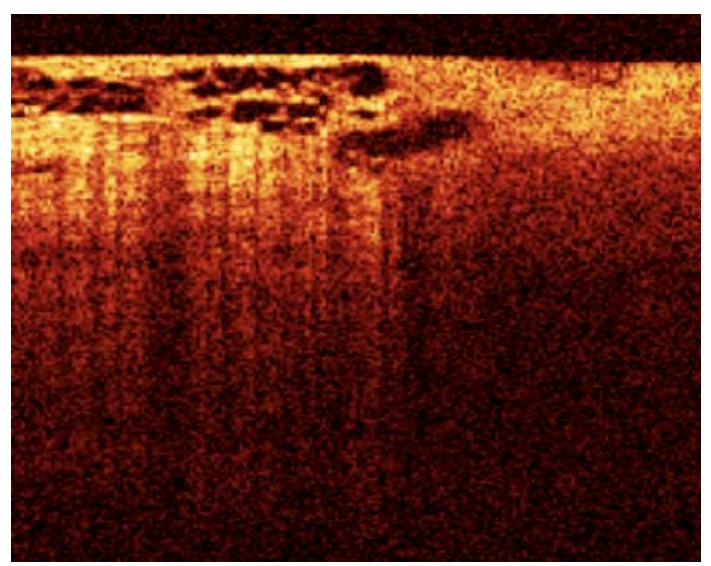

Fig. 7 Optical coherence tomography of lymphatic tissue which may be confused for a neurovascular bundle during prostatectomy established in ophthalmology, its use in urology continues to evolve. With further technological advances in robotic surgery, a synergy between imaging and surgery will provide improved operative results and reduced patient morbidity.

\section{References}

1. Topouzis F, Koskosas A, Pappas T, et al (2007) Foveomacular retinitis and associated optical coherence tomography findings. Ophthalmic Surg Lasers Imaging 38(4):333-335

2. Joeres S, Tsong JW, Updike PG, Collins AT, Dustin L, Walsh AC, Romano PW, Sadda SR (2007) Reproducibility of quantitative optical coherence tomography subanalysis in neovascular agerelated macular degeneration. Invest Ophthalmol Vis Sci 48(9):4300-4307

3. Pitris C, Goodman A, Boppart SA, et al (1999) High-resolution imaging of gynecologic neoplasms using optical coherence tomography. Obstet Gynecol 93:135-139

4. Tearney GJ, Brezinski ME, Southern JF, et al (1997) Optical biopsy in human gastrointestinal tissue using optical coherence tomography. Am J Gastroenterol 92:1800-1804

5. Rollins A, Ung-arunyawee R, Chak A, et al (1999) Real-time in vivo imaging of human gastrointestinal ultrastructure by use of an endoscopic optical coherence tomography with a novel efficient interferometer design. Opt Lett 24:1358

6. Sun CW, Wang YM, Lu LS, Lu CW, Hsu IJ, Tsai MT, Yang CC, Kiang YW, Wu CC (2006) Myocardial tissue characterization based on a polarization-sensitive optical coherence tomography system with an ultrashort pulsed laser. J Biomed Opt. 11(5):054016

7. Gambichler T, Regeniter P, Bechara FG, Orlikov A, Vasa R, Moussa G, Stucker M, Altmeyer P, Hoffmann K (2007) Characterization of benign and malignant melanocytic skin lesions using optical coherence tomography in vivo. J Am Acad Dermatol 57(4):629-637

8. Tearney GJ, Brezinski ME, Southern JF, Bouma BE, Boppart SA, Fujimoto JG (1997) Optical biopsy in human urologic tissue using optical coherence tomography. J Urol 157(5):1915-1919

9. Jesser CA, Boppart SA, Pitris C, et al. (1999) High resolution imaging of transitional cell carcinoma with optical coherence tomography; feasibility for the evaluation of bladder pathology. Br J Radiol 72:1170-1176

10. Manyak MJ, Gladkova ND, Makari JH, et al (2005) Evaluation of superficial bladder transitional-cell carcinoma by optical coherence tomography. J Endod 19(5):570-574

11. Zagaynova EV, Streltsova OS, Gladkova ND, et al (2002) In vivo optical coherence tomography feasibility for bladder disease. J Urol 167:1492-1496

12. Bill-Axelson A, Holmberg L, Ruutu M, Haggman M, et al (2005) Scandinavian prostate cancer group study no. 4. Radical prostatectomy versus watchful waiting in early prostate cancer. N Engl J Med 352:1977-1984

13. Walsh PC (2005) Anatomical radical retropubic prostatectomy: detailed description of the surgical technique. J Urol 173(1):324

14. Boppart SA, Herrmann JM, Pitris C, et al (2001) Real-time optical coherence tomography for minimally invasive imaging of prostate ablation. Comput Aided Surg 6:94-103

15. Aron M, Kaouk JH, Hegarty NJ, et al (2007) Preliminary experience with NIRIS optical coherence tomography system during laparoscopic and robotic prostatectomy. J Endourol 21(8):814818 
16. Fried NM, Rais-Bahrami S, Lagoda GA, Chuang Y, Burnett AL, Su LM (2007) Imaging the cavernous nerves in the rat prostate using optical coherence tomography. Lasers Surg Med 39:36-41

17. Gill IS, Matin SF, Desai MM, et al (2003) Comparative analysis of laparoscopic versus open partial nephrectomy for renal tumors in 200 patients. J Urol 170:64-68
18. Chung B, Tresser N, Kareta K, et al (2006) Niris Optical Coherence Tomography System. Principles of operation and applications in partial nephrectomy. In: Abstract of the 5th international kidney cancer symposium, Chicago 